\title{
Trends of Tuberculosis and Treatment Outcomes at Mizan Aman General Hospital, Southwest Ethiopia: A Four Year Retrospective Study
}

Temesgen Fiseha $^{1 *}$, Teklemichael Gebru ${ }^{1,2}$, Hordofa Gutema ${ }^{2,3}$, Yamrot Debela ${ }^{2,3}$

${ }^{1}$ Department of Clinical Laboratory Science, College of Medicine and Health Science, Wollo University, Dessie, Ethiopia

${ }^{2}$ Department of Epidemiology, College of Health Science, Welkite University, Welkite, Ethiopia

${ }^{3}$ Department of Health Education and promotion, School of Public Health, Bahir Dar University, Bahir Dar, Ethiopia

\begin{abstract}
Background: Tuberculosis (TB) is a serious global public health problem and is the second most common cause of death from infectious disease. The aim of this study was to assess trends of TB and treatment outcomes in the past four years in Mizan Aman General Hospital, Southwestern Ethiopia.

Methods: We analyzed the records of 2150 tuberculosis patients registered at Mizan Aman General Hospital from June 2010 to July 2013. Tuberculosis type and treatment outcome were categorized according to the national tuberculosis control program guideline. Data analysis was performed using the statistical package SPSS for windows, version 20.

Results: From the total of 2150 TB patients registered during the indicated period, $2090(97.2 \%)$ were new cases and $60(2.8 \%)$ were re-treated cases. Of which, $1125(52.3 \%)$ were smear negative pulmonary TB (SNPTB) cases and the rest, $532(24.7 \%)$ and $493(23 \%)$ were smear positive pulmonary TB (SPPTB) and extra pulmonary TB (EPTB) cases respectively. Trends of all forms of TB increased from 363 in 2010 to 634 in 2011, and decreased in the year 2012 (330), and then, increased in the year 2013 (823). Regarding treatment outcomes, 561 (26.1\%) were successfully treated, $26(1.2 \%)$ defaulted, $49(2.3 \%)$ died, and 1514(70.4\%) patients were transferred out. Age and year of enrollment of TB patients were associated with higher treatment success $(P<0.05)$. On the other hand, patient category, HIV status and year of enrollment of TB patients were significantly associated with death rate $(P<0.05)$.
\end{abstract}

Conclusion: TB in the hospital showed a fluctuating pattern for the last four years and the proportion of SNPTB cases was highest compared to cases of SPPTB and EPTB. The treatment success of all types of TB was unsatisfactory, which is a serious public health concern that needs to be addressed.

Keywords: Tuberculosis; Treatment outcomes; Southwestern Ethiopia

\section{Introduction}

Tuberculosis (TB) is the world's second most common cause of death from infectious disease, next to HIV/AIDS. In 2012, there were an estimated 8.6 million incident cases of TB and a total of 1.1 million people died as a result of the disease, most of these occurring in developing countries [1]. The disease which affects both adults and children remains a major cause of mortality despite availability of effective treatment [2]; and if TB controls are not strengthened, the World Health Organization (WHO) estimates that, by 2020, 70 million more people will die from it. More than this, an estimated 1.1 million (13\%) of the 8.6 million people who developed TB in 2012 were HIVpositive; with $75 \%$ of these in the African Region.

Ethiopia, a country with a population of 90 million people, ranks seventh among the 22 high-burden countries (HBCs) for TB in the world. According to the WHO Global TB report 2013, there were an estimated 230,000 TB cases and 16000 deaths in 2012 [1]. The number of TB cases is likely to increase as Ethiopia's HIV/AIDS epidemic expands; among the 272,000 HIV patients screened for TB, $10.2 \%$ were TB positive. Furthermore, the incidence rate of TB is increasing in Ethiopia at a rate of 5 new TB cases per 100.000 populations per year [3].

The incidence of TB cases and mortality from TB are unacceptably large given that most are preventable and curable if people can access health care for a diagnosis and the right treatment is provided. The WHO recommended strategy for global tuberculosis control is a shortcourse, clinically administered treatment. This Directly Observed
Treatment short-course (DOTS) has been found to be an effective means of administering anti-TB drugs, significantly reducing the rates of relapse and drug resistance as well as improving the treatment compliance rate $[4,5]$. In Ethiopia, a standardized TB prevention and control program incorporating DOTS was started as a pilot in 1992, at Arsi zone in Oromia region.

The DOTS strategy ensures that infectious TB patients are identified and cured using standardized drug combination given for at least six to eight months [6]. A successful outcome of treatment for TB, assuming an appropriate drug regimen is prescribed, depends largely on patient adherence to the regimen [7]. Achieving adherence is not an easy task, and many find it difficult to complete their course of treatment and this serves as a major constraint to eradicating the disease $[8,9]$.

In Ethiopia, particularly in Bench Maji zone, information about the epidemiological trends of TB and treatment outcomes by sociodemography, types of $\mathrm{TB}$, and time is minimal. That is to indicate that

*Corresponding author: Temesgen Fiseha, Department of Clinical Laboratory Science, College of Medicine and Health Science, Wollo University, Dessie, Ethiopia, Tel: 033-3115211; E-mail: temafiseha@gmail.com

Received: Decmber 15, 2014; Accepted: December 17, 2014; Published: January 01, 2015

Citation: Fiseha T, Gebru T, Gutema H, Debela Y (2015) Trends of Tuberculosis and Treatment Outcomes at Mizan Aman General Hospital, Southwest Ethiopia: A Four Year Retrospective Study. J Bioengineer \& Biomedical Sci 5: 138. doi:10.4172/2155- 9538.1000138

Copyright: $\odot 2015$ Fiseha T, et al. This is an open-access article distributed under the terms of the Creative Commons Attribution License, which permits unrestricted use, distribution, and reproduction in any medium, provided the original author and source are credited. 
Citation: Fiseha T, Gebru T, Gutema H, Debela Y (2015) Trends of Tuberculosis and Treatment Outcomes at Mizan Aman General Hospital, Southwest Ethiopia: A Four Year Retrospective Study. J Bioengineer \& Biomedical Sci 5: 138. doi:10.4172/2155- 9538.1000138

Page 2 of 5

having summary figures for the zone and analyzing such values will provide information about the zone as a whole and the country in particular. In light of this, the purpose of this study was to examine the trends of TB and treatment outcomes in Mizan Aman General Hospital, Bench Maji zone, southwest Ethiopia [10].

\section{Materials and Methods}

\section{Study location}

The study was conducted at Mizan-Aman General Hospital in Bench Maji Zone Southwest Ethiopia, which is located $561 \mathrm{~km}$ away from Addis Ababa. The hospital is a secondary health care level hospital serving the population of Bench Maji and two adjacent zones. The health facilities within these zones register and treat all diagnosed $\mathrm{TB}$ patients under the DOTS. In the hospital, TB clinic is operating under the National Tuberculosis and Leprosy Program (NTLCP) of Ethiopia, in which patients are diagnosed with TB by examination of morning sputum smears by Zeihel-Nieelsen staining, for the presence of Acid fast bacilli (AFB), chest radiographs, and for EPTB, pathological investigations were used. Patients are referred to the DOTS clinic where they are registered and treated according to the NTLCP. Once diagnosed with $\mathrm{TB}$, patients are provided with anti-TB medication and care free of charge. The hospital provides services for approximately 6679 inpatient and 50386 outpatient attendances a year coming to the hospital from the catchment population of about 1.5 million people. Out of the outpatient attendants the hospital has a total of 1165 registered new cases of TB who came to the TB clinic for follow up, and 404 of them are smear-positive pulmonary TB (SPPTB) patients [11].

\section{Study design and data collection}

A five-year retrospective descriptive analysis to assess trends of $\mathrm{TB}$ and treatment outcomes and risk factors of TB patients registered from august, 2010 to 2014 was carried out in Mizan-Aman General Hospital's TB clinic. All TB patients registered at the TB clinic of the hospital during the study period were included in the study. Because DOTS became nationwide in 2006, retrieval of data was restricted to 2010. The registration document had basic information such as patients' age, sex, type of TB, AFB smear result, treatment regimen, treatment started date, HIV serostatus, and treatment outcomes. Data was collected by trained nurses using structured checklist, and was cheeked on a daily base by the principal investigators. Types of $\mathrm{TB}$ and treatment outcomes were defined according to the standard definitions of the NLCP adopted from WHO [11]. Types of TB was classified as: SPPTB (patient with one or more initial sputum smear examinations, direct smear microscopy, AFB-positive; or one sputum examination AFB positive plus radiographic abnormalities consistent with active pulmonary $\mathrm{TB}$ as determined by a clinician), smear-negative pulmonary TB (SNPTB: patient with pulmonary TB who does not meet the criteria for SPPTB), and Extra-pulmonary TB (EPTB: patient with TB of organs other than the lungs. Patients' treatment outcomes were classified as: cured (finished treatment with negative bacteriology result at the end of the treatment), treatment completed (finished treatment but without bacteriology result at the end of their treatment), defaulted (patients who interrupted their treatment for two consecutive months or more after registration), treatment failure (remaining smear-positive at five months despite correct intake of medication), died (patients who died from any cause during the course of treatment), transferred out (patients whose treatment result is unknown due to transfer to another health facility), and successfully treated (a patient who was cured or completed treatment).

\section{Statistical analysis}

Data were entered in to Epi-data Version 3.1 and were exported in to SPSS Version 20 software package for analysis,. For categorical outcomes, we calculated relative risks from the proportions in each group and tested the differences using the Chi-square test or the Fisher exact test. A P-value $<0.05$ was considered significant.

\section{Ethical considerations}

The study was approved by health research and post graduate coordinating office, college of Public health and medical science of Jimma University. Letter of co-operation was obtained from Bench Maji zone health department and Mizan-Aman General hospital.

\section{Results}

\section{Patient characteristics}

A total of 2150 TB patients were registered and treated with all forms of TB for the past four years. Out of which, 1125 (52.3\%) were SNPTB cases and the rest, $532(24.7 \%)$ and 493 (23\%) were SPPTB and EPTB cases respectively. Among all forms of TB, 1234 (57.4\%) were male patients and $916(42.6 \%)$. TB patients had a mean age of 29 years, ranging from 1 to 90 years. Out of the total TB cases, 397 (18.5\%) were HIV positive; with $52.9 \%$ of males and $85.1 \%$ in the age group of 15 to 44 years. In terms of disease status, 2090 (97.2\%) were new cases and the rest, $56(2.6 \%)$ were relapse cases and $4(0.2 \%)$ were return after defaulting (Table 1).

Table 2 shows trends of all forms of TB cases across the years during the study period. Trends of all forms of TB has increased from 363 in the year 2010 to 634 in the year 2011, then decreased to 330 in 2012 and increased to 823 in the year 2013. The same trends are observed when the data are disaggregated by types of TB: sputum smear positive, smear negative, extrapulmonary TB cases (Figure 1). There are consistently and significantly more SNPTB cases reported each year than SPPTB or EXPTB cases. The least and highest number of annually reported new SPPTB cases over the period were 66 cases in 2012 and 193 cases in 2013, respectively. The cases of all forms of TB were higher among male TB patients $(P=0.005)$. The proportion of

\begin{tabular}{|c|c|c|c|}
\hline Characteristics & & Frequency & Percent \\
\hline \multicolumn{4}{|l|}{ Sex } \\
\hline & Male & 1234 & 57.4 \\
\hline & Female & 916 & 42.6 \\
\hline \multicolumn{4}{|l|}{ Age group } \\
\hline & $0-14$ years & 263 & 12.2 \\
\hline & $15-44$ years & 1587 & 73.8 \\
\hline & $45-64$ years & 260 & 12.1 \\
\hline & $\geq 65$ years & 40 & 1.9 \\
\hline \multicolumn{4}{|c|}{ TB patients category } \\
\hline & New & 2090 & 97.2 \\
\hline & Relapse & 56 & 2.6 \\
\hline & Return after default & 4 & 0.2 \\
\hline \multicolumn{4}{|l|}{ Type of TB } \\
\hline & SPPTB & 532 & 24.7 \\
\hline & SNPTB & 1125 & 52.3 \\
\hline & EXPTB & 492 & 23.0 \\
\hline \multicolumn{4}{|l|}{ HIV status } \\
\hline & Positive & 397 & 18.5 \\
\hline & Negative & 1753 & 81.5 \\
\hline
\end{tabular}

Table 1: Characteristics of TB patients registered at Mizan Aman General Hospital, Southwest Ethiopia, 2010 to 2013 ( $n=2150)$. 
Citation: Fiseha T, Gebru T, Gutema H, Debela Y (2015) Trends of Tuberculosis and Treatment Outcomes at Mizan Aman General Hospital, Southwest Ethiopia: A Four Year Retrospective Study. J Bioengineer \& Biomedical Sci 5: 138. doi:10.4172/2155- 9538.1000138

Page 3 of 5

\begin{tabular}{|c|c|c|c|c|c|c|c|c|}
\hline \multirow[t]{2}{*}{ Years } & \multicolumn{4}{|c|}{ New cases } & \multicolumn{3}{|c|}{ Re-treated cases } & \multirow[t]{2}{*}{ All TB Cases } \\
\hline & $\begin{array}{l}\text { SPPTB } \\
\text { N (\%) }\end{array}$ & $\begin{array}{l}\text { SNPTB } \\
\mathbf{N}(\%)\end{array}$ & $\begin{array}{l}\text { EXPTB } \\
\text { N (\%) }\end{array}$ & Total N (\%) & $\begin{array}{l}\text { Relapse } \\
\text { N (\%) }\end{array}$ & $\begin{array}{l}\text { Return after } \\
\text { default N (\%) }\end{array}$ & $\begin{array}{l}\text { Total } \\
\text { N (\%) }\end{array}$ & \\
\hline 2010 & $79(21.9)$ & $200(55.6)$ & $81(22.5)$ & $360(99.2)$ & $3(100)$ & $0(0)$ & $3(0.8)$ & 363 \\
\hline 2011 & $167(26.9)$ & $309(49.8)$ & $145(23.3)$ & $621(97.9)$ & $12(92.3)$ & $1(6.7)$ & $13(2.1)$ & 634 \\
\hline 2012 & $66(20.7)$ & $171(53.6)$ & $82(25.7)$ & $319(96.7)$ & $10(90.9)$ & $1(9.1)$ & $11(3.3)$ & 330 \\
\hline 2013 & $193(24.4)$ & $419(53.0)$ & $178(22.5)$ & $790(96.0)$ & $31(93.9)$ & $2(6.1)$ & $33(4.0)$ & 823 \\
\hline Total & $505(24.2)$ & $1099(52.6)$ & $486(23.2)$ & $2090(97.2)$ & $56(93.3)$ & $4(6.7)$ & $60(2.8)$ & 2150 \\
\hline
\end{tabular}

Table 2: Trends of all forms of TB cases registered in Mizan Aman General Hospital, Southwest Ethiopia, 2010- 2013 ( $n=2150$ ).

\begin{tabular}{|c|c|c|c|c|c|c|c|}
\hline \multirow[t]{2}{*}{ Years } & \multirow[t]{2}{*}{ Disease status } & \multicolumn{6}{|c|}{ Treatment outcomes } \\
\hline & & C N (\%) & TC N (\%) & De N (\%) & D N (\%) & TO N (\%) & TS N (\%) \\
\hline \multirow[t]{3}{*}{2010} & New & $32(8.9)$ & $116(32.2)$ & $9(2.5)$ & $21(5.8)$ & $182(50.6)$ & $148(41.1)$ \\
\hline & Re-treated & $1(33.3)$ & - & - & $1(33.3)$ & $1(33.3)$ & $1(33.3)$ \\
\hline & Total & $33(9.1)$ & $116(32.0)$ & $9(2.5)$ & $22(6.1)$ & $183(50.4)$ & $149(41.0)$ \\
\hline \multirow[t]{3}{*}{2011} & New & $31(5.0)$ & $95(15.3)$ & $7(1.1)$ & $6(1.0)$ & $482(77.6)$ & $126(20.3)$ \\
\hline & Re-treated & $2(15.4)$ & $2(15.4)$ & $1(7.7)$ & $1(7.7)$ & $7(53.8)$ & $4(30.8)$ \\
\hline & Total & $33(5.2)$ & 97 (15.3) & $8(1.3)$ & $7(1.1)$ & $489(77.1)$ & $130(20.5)$ \\
\hline \multirow[t]{3}{*}{2012} & New & 37 (11.6) & $102(32.0)$ & $3(0.9)$ & $11(3.4)$ & $166(52.0)$ & $139(43.6)$ \\
\hline & Re-treated & $4(36.4)$ & $5(45.5)$ & - & $1(9.1)$ & $1(9.1)$ & 9 (81.8) \\
\hline & Total & $41(12.4)$ & $107(32.4)$ & $3(0.9)$ & $12(3.6)$ & $167(50.6)$ & $148(44.8)$ \\
\hline \multirow[t]{3}{*}{2013} & New & $17(2.2)$ & $110(13.9)$ & $6(0.8)$ & $6(0.8)$ & $651(82.4)$ & $127(16.1)$ \\
\hline & Re-treated & $1(3.0)$ & $6(18.2)$ & - & $2(6.1)$ & $24(72.7)$ & $7(21.2)$ \\
\hline & Total & $18(2.2)$ & $116(14.1)$ & $6(0.7)$ & $8(1.0)$ & $675(82.0)$ & $134(16.3)$ \\
\hline \multicolumn{2}{|l|}{ Total } & $125(5.8)$ & $436(20.3)$ & $26(1.2)$ & $49(2.3)$ & $1514(70.4)$ & $2150(100)$ \\
\hline
\end{tabular}

$C=$ cured, $T C=$ treatment completed, $D e=$ defaulted, $D=$ died, TO transfer out, $T S=$ treatment success

Table 3: Treatment outcomes of all forms of TB cases registered in Mizan Aman General Hospital, Southwest Ethiopia, 2010 to 2013 ( $n=2150$ ).

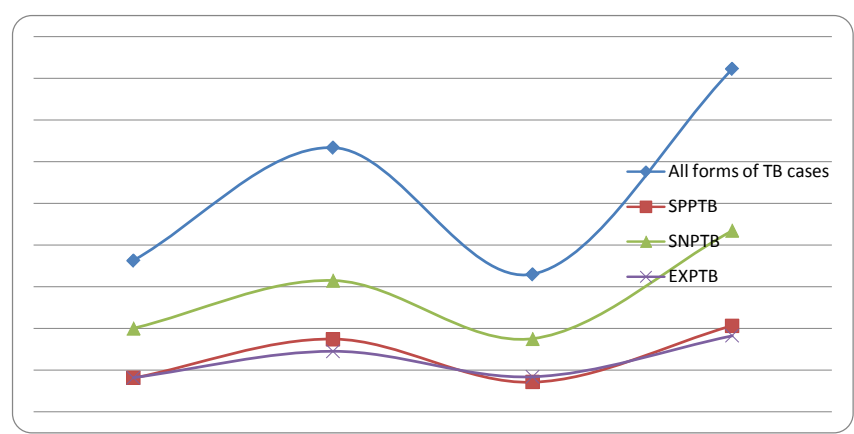

Figure 1: Trends of TB across the years in Mizan Aman general hospital, 20102013.

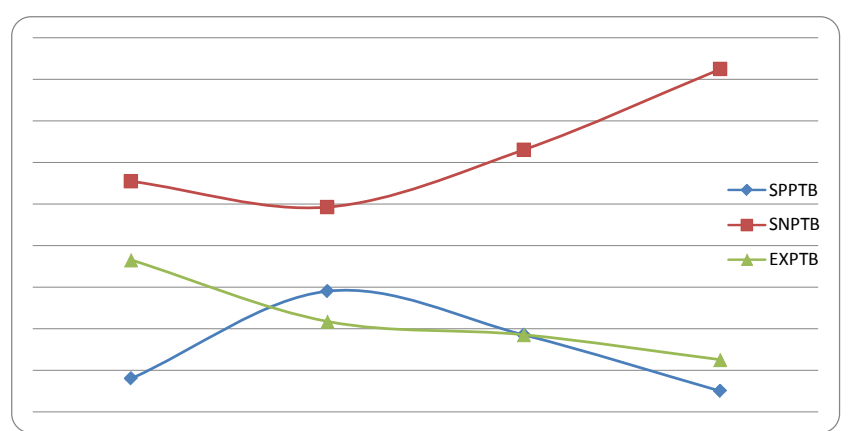

Figure 2: Trends of all forms of TB by age group in Mizan Aman general hospital, 2010-2013.

EPTB showed a decreasing trend as the age of patients' increased; with $36.5 \%, 21.7 \%, 18.5 \%$, and $12.5 \%$ among those with $0-14,15-44,45-64$, and $\geq 65$ years respectively (Figure 2 ).

From a total of 2150 tuberculosis patients analyzed from July 2010 to June 2013: 125 (5.8\%) cured, 436 (20.3\%) completed treatment, 26 (1.2\%) defaulted, 49 (2.3\%) died, and $1514(70.4 \%)$ were transferred out to other health facility. The proportion of treatment success was $26.1 \%(561 / 2150)$ among all types of TB patients without significant gender effect $(26.1 \%$ among males and $26.1 \%$ among females; $P>0.05)$. The treatment success was $29.1 \%$ among SPPTB patients, $24.5 \%$ among SNPTB, and $26.4 \%$ among EXPTB patients.

The treatment success varies from $26.6 \%$ in 2010 to $23.2 \%$ in the year 2011. The cure rate of TB patients increased from $33(26.4 \%)$ in 2010 and 2011 to 41 (32.8\%) in 2012, and then decreased to $18(14.4 \%)$ in 2013. After 2010, the rate of treatment completion for all forms of TB cases increased markedly. Default rate was steadily decreased across years from $9(34.6 \%)$ in 2010 to $3(11.5 \%)$ in 2012, then increased to 6 $(23.1 \%)$ in 2013 . On the other hand, the rate of death fluctuated across the period, with $22(44.9 \%)$ in 2010, 7 (14.3\%) in 2011, $12(24.5 \%)$ in 2012 and $8(16.3 \%)$ in 2013 (Table 3).

Age and year of enrollment of TB patients were associated with higher treatment success rate $(P<0.05)$. However, disease status, age, HIV status of TB patients and year of enrollment were significantly associated with potentially unsuccessful outcome compared to treatment success $(P<0.05)$. On the other hound, disease status, HIV status of TB patients and year of enrollment were significantly associated with death rate $(P<0.05)$.

\section{Discussion}

In this health institution based retrospective study, information on the overall trend of TB and treatment outcome across the year during the study period were assessed in Mizan Aman general hospital, Bench 
Maji Zone, southern Ethiopia. The trends in all types of TB in this study showed a fluctuating pattern from year to year, which might be as the result of inconsistency in prevention and control program and health professional turnover and as a result of high population movement in the zone associated with gold mining. The proportions of the different types of TB recorded in were similar with those reported in Gonder University Teaching hospital [12], but different proportions were reported from Gambella regional hospital [13].

The high proportion of SPPTB observed among male TB patients in this study was consistent with the study conducted in Gambella regional hospital [13] and the epidemiological analysis of TB trends in Ethiopia [3], in which men are disproportionately affected by TB. Age groups of 0-14 years were affected more with EPTB than the other age groups across the years during the study period. This is in agreement with the reports of the study in Gambella in which EPTB showed a trend of decreasing as the age of patients' increased with highest observed in the age group of 0-14 years [13].

In this study, the proportion of HIV co-infection among TB patients was $18.5 \%$; nearly similar to the studies conducted in Nigeria $(20.0 \%)$ [14]. However, this finding is lower than that of study conducted in Northwest Ethiopia (25\%) [15], and higher than that of 2013 WHO report for Ethiopia (9.6\%). This high prevalence of HIV co-infection among TB patients in the study area signifies the urgent need for staff capacity building and increasing public awareness.

The present study found that the successful treatment rate of all TB cases 561 (26.1\%) treated at the TB clinic of Mizan Aman General Hospital was unsatisfactory when compared to the 2011 WHO report on global TB control, were the TSR among 22-high burden countries varied from $65 \%$ in the Russian Federation to $95 \%$ in China [1]. Likewise, the target set in Ethiopia HSDP III for the prevention and control of TB was to achieve $85 \%$ TSR [16] that was very high compared to our finding. The low TSR observed in this study might be due to high transferred out rate $(70.4 \%)$, were treatment outcome of patients who were transferred out were unknown.

Studies conducted in Felege Hiwot Referral Hospital and Gonder University Teaching hospitals, in northwest Ethiopia reported TSR $26 \%$ and $29.5 \%$ of all TB cases, respectively which are comparable to the findings of our study $[12,15]$. The higher proportion of treatment success rate among TB patients observed among younger age group in this study; may be due to their increased family, organizational, and societal responsibilities as people in this age group involve themselves in various extraneous daily activities in order to win the socioeconomic hardship which increases the frequency of their contact with health facility.

As in Istanbul, Turkey study, which showed that older age group patients were at higher risk for adverse treatment outcome [17], in our study, being older age group was associated with unsuccessful treatment outcome. This may be partly explained due to co-infection and general physiological deterioration with age, and thus it is crucial to monitor TB treatment in older patient. Furthermore, being HIV-positive was associated with unsuccessful treatment outcome. According to WHO's statistics, treatment outcomes are worse among HIV-positive TB patients compared with HIV-negative TB patients [1]. Likewise, some studies showed that HIV co-infection was associated with an increased risk for unsuccessful treatment outcome among TB cases [14].

The default rate in this study (1.2\%) was lower than the average $6.2 \%$ observed among the 22 HBCs [18], and other studies conducted in the country; $22.9 \%, 18.3 \%$ and $2.5 \%$ in Gambella, Gondar and Felege
Hiwot hospitals, respectively $[12,13,15]$. This lower defaulter rate in this study might be due to proper supervision and health education in the study area. In agreement with the study conducted in Gondar hospital, this study also found a significantly decreasing death rate of TB patients across the years from $6.1 \%$ in 2010 to $1.0 \%$ in 2013 $(\mathrm{P}<0.001)$. Furthermore, the death rate of TB patients was significantly higher among TB-HIV co-infection (5.5\% vs. $1.5 \%$; $\mathrm{P}<0.001)$. Similar finding was reported from the study conducted in Felege Hiwot Referral Hospital, northwest Ethiopia; indicating that special attention to HIV-positive TB cases is required in the study area.

\section{Conclusion}

Trends in annual number of TB cases had showed a fluctuating pattern for the last for years, and the proportion of HIV co-infection among TB patients was $18.5 \%$, which is a serious public health concern that needs to be addressed right away. The TSR of all forms of TB patients treated at Mizan Aman General Hospital was unsatisfactory (26.1\%) compared to $85 \%$ treatment success suggested by WHO as a threshold. Thus, to improve treatment outcome of tuberculosis patients improved counseling during the intensive and continuation phases of treatment and health education to reduce treatment interruption is required in our setting.

\section{Authors' Contributions}

TF and TG was the primary researcher, conceived the study, designed, participated in data collection, conducted data analysis and drafted the manuscript for publication. HG and YD assisted in data collection, interpretation of the results and preparation of first draft of manuscript. All authors read and approved the final manuscript.

\section{Acknowledgements}

Authors would like to thank all the staff members of TB clinic, Mizan Aman General Hospital for the proper documentation of patient's information and treatment outcome and for their genuine assistance during data collection.

\section{References}

1. World Health Organization (2013) Global tuberculosis report

2. Burton N, Forson A, Lurie M, Kudzawu S, Kwarteng E, et al. (2011) Factors associated with mortality and default among patients with tuberculosis attending a teaching hospital clinic in accra, ghana. Trans R Soc Trop Med Hyg 105: 675-682.

3. Nigatu T, Abraha M (2010) Epidemiological analysis of tuberculosis trends in EthiopiaTüberküloz 58: 375-384.

4. Erhabor GE, Adewole O, Adisa AO, Olajolo OA (2003) Directly observed short course therapy for tuberculosis. A preliminary report of a three-year experience in a teaching hospital 95: 1082-1088.

5. Estifanos B Shargie, Bernt Lindtjorn (2005) DOTS improves treatment outcomes and service coverage for tuberculosis in South Ethiopia: a retrospective trend analysis, BMC Public Health 5.

6. World Health Organization Report (2008) Global Tuberculosis Control, surveillance, planning and financing, Geneva, Switzerland.

7. Volmink J, Garner P(2009) Directly observed therapy for treating tuberculosis Cochrane Collab.

8. International Standards for Tuberculosis Care

9. American Thoracic Society (2003) Centers for Disease Control and Prevention Infectious Diseases Society of America. Treatment of tuberculosis 167: 603662

10. Fedaral Ministry of Health Ethiopia (2008) Tuberculosis, Leprosy and TB/HIV prevention and control programme manual.

11. Ministry of Health of Ethiopia (2009) Tuberculosis, Leprosy and TB/HIV Prevention and Control Programme Manual, Addis Ababa. 
Citation: Fiseha T, Gebru T, Gutema H, Debela Y (2015) Trends of Tuberculosis and Treatment Outcomes at Mizan Aman General Hospital, Southwest Ethiopia: A Four Year Retrospective Study. J Bioengineer \& Biomedical Sci 5: 138. doi:10.4172/2155- 9538.1000138

12. Tessema B, Muche A, Bekele A, Reissig D, Emmrich F et al. (2009) Treatment outcome of tuberculosis patients at Gondar University Teaching Hospital, Northwest Ethiopia. A five-year retrospective study, BMC Public Health 9.

13. Demeke D, Legesse M, Bati J (2013) Trend of Tuberculosis and Treatment Outcomes in Gambella Region with Special Emphasize on Gambella Regional Hospital, Western Ethiopia 3.

14. Babatunde O, Elegbede O, Ayodele M, Fadare J, Isinjaye A, et al. (2013) Factors Affecting Treatment Outcomes of Tuberculosis in a Tertiary Health Center in Southwestern Nigeria. Int Rev Soc Sci Humanit 4: 209-218.
15. Biadglegne F, Anagaw B, Debebe T, Anagaw B, Tesfaye W, et al. (2013) A retrospective study on the outcomes of tuberculosis treatment in Felege Hiwot Referral Hospital, Northwest Ethiopia. Int J Med Med Sci 5: 85-91.

16. Ministry of Health of Ethiopia (2008) Tuberculosis, Leprosy and TB/HIV Prevention and Control Programme Manual, Addis Ababa.

17. Babalık A, Kılıçaslan Z, Kızıltaş Ş, Gencer S, Öngen G (2013) A Retrospective Case-Control Study, Factors Affecting Treatment Outcomes for Pulmonary Tuberculosis in İstanbul, Turkey. Balk Med J 30: 204-210.

18. World Health Organization (2010) The five elements of DOTS, WHO report 2010. Geneva Switz WHO 